\title{
COLLISIONAL PROCESSES IN STELLAR SYSTEMS
}

\author{
IRA H. GILBERT \\ Dept. of Physics, Brandeis University, Waltham, Mass. 02154, U.S.A.
}

\begin{abstract}
The theory of collisional relaxation in stellar systems is discussed in terms of an expansion in powers of $1 / N$, the inverse of the total number of stars. The results are expressed in terms of the concept of gravitational polarization.
\end{abstract}

\section{Probability in Stellar Dynamics}

At first glance stellar dynamics appears to be a remarkably simple subject. One deals with well defined physical systems, collections of idealized point masses, interacting according to the laws of classical mechanics and Newtonian gravitation. One need only prescribe the initial state of the system and integrate the equations of motion in order to determine the state at any future time. The catch, of course, is that the integration of these equations is totally impractical when the number of stars is large enough to represent a real system. Furthermore, the information contained in the precise dynamical state of the system is too detailed to be useful.

The way around these difficulties lies in replacing certainty by chance, i.e. by settling for a description in terms of probabilities instead of precise dynamical states. The general idea is that a statistical description should be simpler and involve fewer variables than an exact one. Furthermore, as the number of stars grows increasingly large the statistical description should improve in accuracy as statistical fluctuations diminish according to the law of large numbers.

Although there is universal agreement on how probabilities are to be handled mathematically there are differences as to their meaning. In my opinion probabilities are introduced in stellar dynamics to represent states of partial knowledge. From this point of view their time dependence, governed by the Liouville equation, reflects the evolution of one's information concerning the dynamical state of the system. If one is provided with some imprecise description of an initial state the Liouville equation allows one to make best possible guesses about the resultant state at some future time.

\section{Alternative Statistical Descriptions}

Let us represent the Liouville probability density for a system of $N$ stars by $D(1,2, \ldots, N)$, where the argument $i$ represents both the position and velocity of particle $i$. In this compact notation $D(1, \ldots, N) \mathrm{d}(1) \mathrm{d}(2) \ldots \mathrm{d}(N)$ is the probability of finding particle 1 within $d \mathbf{r}_{1}$ of position $\mathbf{r}_{1}$ and within $d \mathbf{v}_{1}$ of velocity $\mathbf{v}_{1}$ etc. For simplicity it is convenient to take $D$ to be symmetric in the various stars. If the stars are equally massive this presents no difficulty. If they are not we may still construct a symmetric function by considering the mass of each star to be a random variable. 
Of course each mass, once chosen, remains constant in time. With this extension, the argument $(i)$ represents the position, velocity, and mass of the $i$ th star.

The Liouville equation, a partial differential equation in $6 N+1$ independent variables, is not any easier to solve than the original dynamical equations. The problem is that the Liouville probability density still contains too much information. To obtain a less detailed description one may integrate $D$ over the phase space of all but $s$ particles and so obtain $f^{(s)}$, the $s$-particle distribution function.

$$
f^{(s)}(1, \ldots, s)=\int D(1, \ldots, N) \mathrm{d}(s+1) \ldots \mathrm{d}(N) .
$$

This quantity gives the probability distribution for the coordinates and velocities of a set of $s$ stars, averaged over all possible states of the remaining stars. In particular, the single-particle function,

$$
f(1)=\int D(1, \ldots, N) \mathrm{d}(2) \ldots \mathrm{d}(N),
$$

which will be written for simplicity without a superscript, is the quantity of central interest in stellar dynamics. It is the least detailed of the distribution functions, depending on the coordinates and velocities of a single star. The higher order distribution functions provide increasingly detailed descriptions. Notice that if $s>s^{\prime}, f^{(s)}$ contains all the information in $f^{\left(s^{\prime}\right)}$ and more. Indeed, integrating Equation (1) over the phase space of stars $s^{\prime}+1$ through $s$ yields the identity

$$
f^{\left(s^{\prime}\right)}\left(1, \ldots, s^{\prime}\right)=\int f^{(s)}(1, \ldots, s) \mathrm{d}\left(s^{\prime}+1\right) \ldots \mathrm{d} s,
$$

which connects these functions.

Now the original idea in introducing probabilities was to simplify the problem by ignoring the finer details while retaining the main macroscopic features. The lower order distribution functions do just this. If we can determine $f$ as a function of the time we will have achieved our goal. The trouble, however, is that the equation which determines the time dependence of $f$ turns out to involve $f^{(2)}$. Quite generally, the equation for $f^{(s)}$ involves $f^{(s+1)}$. The entire set of equations, called the BBGKY hierarchy for Bogolioubov, Born, Green, Kirkwood, and Yvon, are therefore coupled. In order to solve for $f$ without solving for all the distribution functions at one time which would again be equivalent to integrating the original equations of motion) these equations must be decoupled. This is accomplished with the aid of certain combinations of the distribution functions called correlation functions. They are symbolized by $g^{(s)}(1, \ldots, s) s=2, \ldots, N$, and defined by equations of the form

$$
\begin{aligned}
f^{(2)}(1,2)= & f(1) f(2)+g(1,2), \\
f^{(3)}(1,2,3)= & f(1) f(2) f(3)+f(1) g(2,3)+f(2) g(1,3)+ \\
& +f(3) g(1,2)+g^{(3)}(1,2,3),
\end{aligned}
$$

where for simplicity the superscript of the two-particle correlation function, which appears frequently in the following discussion, has been suppressed. In general $f^{(s)}$ 
is expressed as a sum of products, each corresponding to a different partition of the integers 1 through $s$.

Unlike the $f$ 's the $g$ 's are essentially independent. Equation (3), expressed in terms of the $g$ 's, becomes

$$
\int g^{(s)}(1, \ldots s) \mathrm{d}(s)=0 \quad s=2, \ldots, N .
$$

It no longer connects different functions but constrains each correlation function separately. The only reason the various $g$ 's are not completely independent is that the distribution functions, being probability densities, are non-negative. This means that the $g$ 's must satisfy a set of inequalities such as

$$
f(1) f(2)+g(1,2) \geqslant 0 \text {, }
$$

etc.

\section{Correlations, Dynamics, and Initial Conditions}

The correlation functions, as their name would indicate, represent multi-particle correlations. If one independently distributes stars according to some single-particle distribution function all of the $g$ 's will vanish. On the other hand, if one independently distributes pairs of stars $g^{(2)}$ will not necessarily vanish but all higher order correlation functions will.

Statistical correlations between stars are determined both by the initial probability distribution and the gravitational dynamics. It seems plausible that in most cases the disorganized motions of the stars will disrupt groups which were initially nearby and quickly erase the initial correlations. The correlations which result will be determined by the dynamics and the single-particle distribution function alone. In order to obtain a closed theory in which the single-particle function is the only variable we will assume this to be the case. More precisely, we will assume probability distributions which have evolved from initially uncorrelated states.

It is, however, possible to imagine situations which are quite different. For example, if one randomly distributes tightly bound binaries it will take a very long time for encounters to disrupt them and so eliminate the initial correlations. We will not consider this possibility.

\section{Expansion in Powers of $1 / N$}

Up to this point we have not used the important piece of information that $N$ is a large number. It is just this fact, however that results in the decoupling of the BBGKY hierarchy which was the reason for introducing the correlation functions in the first place.

The Liouville equation involves $N$ both explicitly, through the summations that appear, and implicitly, through the interstellar force. This implicit dependence comes about because we are considering $M$, the total mass of the system, to be fixed and therefore the individual stellar masses to be proportional to $1 / N$. For simplicity 
assume all the stars to have the same mass, $M / N$. The Liouville equation may then be written

where

$$
\frac{\partial D}{\partial t}+\sum_{i=1}^{N} \mathbf{v}_{i} \cdot \frac{\partial}{\partial \mathbf{r}_{i}} D+\frac{1}{N} \sum_{i, j=1}^{N} \mathbf{a}_{i, j} \cdot \frac{\partial}{\partial \mathbf{v}_{j}} D=0,
$$

$$
\frac{1}{N} \mathbf{a}_{i, j}=-\frac{M G}{N} \frac{\left(\mathbf{r}_{i}-\mathbf{r}_{j}\right)}{\left|\mathbf{r}_{i}-\mathbf{r}_{j}\right|^{3}}
$$

is the acceleration of star $i$ due to star $j$. All dependence on $N$ is now explicit.

A set of equations for the correlation functions may be obtained from the Liouville equation in a number of ways, the simplest being by means of a generating functional first introduced by Bogolioubov. The advantage of this technique is that the single functional equation that results is entirely equivalent to the set of $N$ differential equations for the $g$ 's. This makes it easy to establish certain general results.

It turns out that there are solutions of these equations for which $g^{(s)}$ is of order $(1 / N)^{s-1}$ for all $s$. Thus if one starts with initial correlations with this dependence on $N$ the dependence is maintained by the dynamics. In particular, if one starts from an initially uncorrelated state, as previously discussed, then at later times $g^{(s)}$ will be of order $(1 / N)^{s-1}$.

The mean total acceleration at $\mathbf{r}_{1}$ is obtained by averaging $\mathbf{a}_{1,2} / N$, the acceleration due to a single star at $\mathbf{r}_{2}$, over all possible values of $\mathbf{r}_{2}$ and then multiplying by $N$.

$$
\mathbf{A}_{1}=\int \mathbf{a}_{1,2} f(2) \mathrm{d}(2)
$$

The first two equations for the correlation functions turn out to be:

$$
\frac{\partial f(1)}{\partial t}+\mathbf{v}_{1} \cdot \frac{\partial}{\partial \mathbf{r}_{1}} f(1)+\frac{N-1}{N} \mathbf{A}_{1} \cdot \frac{\partial}{\partial \mathbf{v}_{1}} f(1)=-\frac{\partial}{\partial \mathbf{v}_{1}} \cdot \int \mathbf{a}_{1,2} g(1,2) \mathrm{d}(2),
$$

and

$$
\begin{aligned}
\frac{\partial g(1,2)}{\partial t}+ & \left(\mathbf{v}_{1} \cdot \frac{\partial}{\partial \mathbf{r}_{1}}+\mathbf{v}_{2} \cdot \frac{\partial}{\partial \mathbf{r}_{2}}\right) g(1,2)+\frac{N-2}{N}\left(\mathbf{A}_{1} \cdot \frac{\partial}{\partial \mathbf{v}_{1}}+\mathbf{A}_{2} \cdot \frac{\partial}{\partial \mathbf{v}_{2}}\right) \times \\
& \times g(1,2)+\frac{N-2}{N}\left(\frac{\partial f(1)}{\partial \mathbf{v}_{1}} \cdot \int \mathbf{a}_{1,3} g(2,3) \mathrm{d}(3)+\frac{\partial f(2)}{\partial \mathbf{v}_{2}} \times\right. \\
& \left.\times \int \mathbf{a}_{2,3} g(1,3) \mathrm{d}(3)\right)+\frac{1}{N}\left[\left(\mathbf{a}_{1,2}-\mathbf{A}_{1}\right) \cdot \frac{\partial}{\partial \mathbf{v}_{1}}+\right. \\
& \left.+\left(\mathbf{a}_{2,1}-\mathbf{A}_{2}\right) \cdot \frac{\partial}{\partial \mathbf{v}_{2}}\right] f(1) f(2) \\
& +\frac{1}{N}\left(\mathbf{a}_{1,2} \cdot \frac{\partial}{\partial \mathbf{v}_{1}}+\mathbf{a}_{2,1} \cdot \frac{\partial}{\partial \mathbf{v}_{2}}\right) g(1,2)
\end{aligned}
$$




$$
\begin{aligned}
& +\frac{1}{N}\left(f(2) \frac{\partial}{\partial \mathbf{v}_{1}} \cdot \int \mathbf{a}_{1,3} g(1,3) \mathrm{d}(3)+f(1) \frac{\mu}{\partial \mathbf{v}_{2}} \times\right. \\
& \left.\times \int \mathbf{a}_{2,3} g(2,3) \mathrm{d}(3)\right)+\frac{N-2}{N} \int\left(\mathbf{a}_{1,3} \times\right. \\
& \left.\times \frac{\partial}{\partial \mathbf{v}_{1}}+\mathbf{a}_{2,3} \cdot \frac{\partial}{\partial \mathbf{v}_{2}}\right) g^{(3)}(1,2,3) \mathrm{d}(3)=0 .
\end{aligned}
$$

These equations are horribly complicated but exact. Equation (10) may be simplified by taking advantage of the smallness of $1 / N$ and neglecting terms of higher order than the first. Recalling that $g^{(s)}$ is of order $(1 / N)^{s-1}$ we see that the last three lines of the equation may be dropped and the factor $N-2 / N$ replaced by unity. We find

$$
\begin{aligned}
\frac{\partial g(1,2)}{\partial t}+ & \left(\mathbf{v}_{1} \cdot \frac{\partial}{\partial \mathbf{r}_{1}}+\mathbf{v}_{2} \cdot \frac{\partial}{\partial \mathbf{r}_{2}}+\mathbf{A}_{1} \cdot \frac{\partial}{\partial \mathbf{v}_{1}}+\mathbf{A}_{2} \cdot \frac{\partial}{\partial \mathbf{v}_{2}}\right) g(1,2) \\
& +\frac{\partial f(1)}{\partial \mathbf{v}_{1}} \cdot \int \mathbf{a}_{1,3} g(2,3) \mathrm{d}(3)+\frac{\partial f(2)}{\partial \mathbf{v}_{2}} \cdot \int \mathbf{a}_{2,3} g(1,3) d(3) \\
& +\frac{1}{N}\left[\left(\mathbf{a}_{1,2}-\mathbf{A}_{1}\right) \cdot \frac{\partial}{\partial \mathbf{v}_{1}}+\left(\mathbf{a}_{2,1}-\mathbf{A}_{2}\right) \cdot \frac{\partial}{\partial \mathbf{v}_{2}}\right] f(1) f(2)=0 .
\end{aligned}
$$

Equations (9) and (11) serve to determine $f$ and $g$ and so achieve the desired closed description in terms of low order distribution functions.

We may note that these equations are similar but not identical to the corresponding equations of plasma physics. There, the factor $N-1 / N$ does not appear in the equation for $f$ and the inhomogeneous term in the equation for $g$ does not involve $A_{i}$. This difference comes about because the plasma equations are based upon an expansion in powers of the inverse of the number of electrons in a sphere whose radius is the Debye length. This number is independent of the total number of electrons in the system, usually taken as infinite. On the other hand the analog of the Debye length for a stellar system such as a globular cluster is the radius of the system itself. Thus, in a stellar system the number of stars in a Debye sphere and the total number of stars are necessarily equal and $N$, as it appears here, has a dual significance.

\section{The Kinetic Equation}

Equations (9) and (11) may, in principle, be simultaneously solved for $f$ and $g$ as functions of the time. The simplest situation occurs when the system is in equilibrium with respect to purely collective motions and the only time dependence is through the slow, secular, effects of collisions. In that case the zeroth order terms in Equation (9) vanish and $\partial f / \partial t$ is seen to be of first order in $1 / N$. It is then reasonable to assume that $\partial g / \partial t$ will be of second order allowing us to drop the first term in Equation (11). This equa- 
tion then serves to determine $g$ as a functional of $f$ which, when inserted into the right hand side of Equation (9) yields a kinetic equation for $f$ alone.

The actual elimination of $g$ may be explicitly carried out for homogeneous plasmas because the spatial homogeneity allows the use of the spatial Fourier transform, resulting in an essentially algebraic relation between $f$ and $g$. The result is the BalescuLenard equation. In stellar systems, which are necessarily inhomogeneous, this technique fails. Although an explicit elimination of $g$ in terms of $f$ has not been achieved it is still possible to construct a formal solution and to interpret it in terms of the underlying physical processes.

\section{Gravitational Polarization}

The physical content of the formal solution previously mentioned is most easily understood in terms of the auxiliarly concept of gravitational polarization. This quantity represents the response of the system to the gravitational field of a selected star moving in a specified orbit. In calculating this response one ignores collisional effects entirely and treats the field of the selected star as a small, externally applied perturbation. The polarization is the change in the single-particle distribution function that this perturbation induces. Symbolized by $\mathbf{f}(1 / 2)$ it represents the change in the probability density for finding a star at $\mathbf{r}_{1}$ with velocity $\mathbf{v}_{1}$ given that another star is known to be located at $\mathbf{r}_{2}$ and moving with velocity $\mathbf{v}_{2}$.

There is a very interesting relation, first noted by Rostoker, between the polarization and the two-particle correlation function. It is

$$
g(1,2)=\tilde{f}(2 / 1) f(1)+\tilde{f}(1 / 2) f(2)+N \int \tilde{f}(1 / 3) \tilde{f}(2 / 3) f(3) \mathrm{d}(3)
$$

The final result of this analysis is that collisional effects in stellar systems, i.e. dynamical effects of order $1 / N$, may be divided into two distinct processes. The first is the gravitational force exerted on each star by the polarization it induces. This may be termed 'polarization drag'. The second is the effect upon each star of the random, fluctuating field resulting from the superposition of the fields of the other stars, each modified by its own polarization. These stars are to be considered to move in unperturbed orbits and not to respond to the influence of the field star under consideration. This last effect may be termed statistical acceleration.

The effects of these two kinds of processes are quite different. The statistical acceleration of a star will, on the average, increase its energy. Because of the identity of inertial and gravitational mass the statistical acceleration affects all stars in the same way. On the other hand, since the polarization induced by a given star is proportional to its mass we may expect heavy stars to be slowed more effectively by polarization drag than light ones. The competition between polarization and statistical effects should lead to a relative concentration of heavier stars in the central portions of a stellar system and lighter ones in the outer portions, i.e. an approach to equipartition. 


\section{Divergences}

The theory sketched here completely takes into account the effects of collective interactions and spatial inhomogeneity which are absent from more elementary treatments. As a consequence, no long-range divergence appears as it does in the Fokker-Planck theory. It is interesting that although the equations of plasma physics and stellar dynamics are very similar, the mechanism for the elimination of this divergence is different in the two cases.

In plasmas the repulsive interparticle force results in Debye shielding which cuts the force off, eliminating the divergence. In stellar systems the attractive interstellar force results in 'anti-shielding' or amplification of the bare gravitational force of a star. This tends to make the divergence worse. It is only the limited spatial extent of the system that finally removes it.

There is, however, in the present theory a divergence at small distances. It comes about because the expansion in powers of $1 / N$ is non-uniformly convergent. The physics behind this is quite simple.

In simplifying Equation (10) we assumed $g$ to be small compared with $f$. But $g$ actually measures the deviations of stars from the paths they would follow if subject only to the mean field. Thus, when two stars approach so closely that they are substantially deflected the assumption that $g$ is small breaks down. It turns out that Equation (11) yields accurate values for $g$ only for interstellar distances which are large compared with the impact parameter for $90^{\circ}$ scattering. As this distance goes to zero the equation yields an unphysical infinity.

This divergence is precisely equivalent to the one occurring in elementary FokkerPlanck treatments in which the momentum transferred in a collision is approximated by integrating the force exerted by one particle upon another while assuming them to move in straight lines. At vanishingly small impact parameters one then erroneously calculates infinite momentum transfer. One can eliminate this error simply by replacing the straight line approximation by the correct two-body orbits.

In the present theory the rigorous treatment of two-body dynamics requires retaining all terms in $g$ appearing in Equation (10). The term in $g^{(3)}$ may still be dropped because it is important only for collisions in which three stars are simultaneously deflected through large angles. The result is again a closed set of equations for $f$ and $g$. This method of eliminating the divergence in $g$ is, however, quite complicated. A simpler approach seems preferable. One merely replaces the true inverse-square force by a fictitious force carefully chosen so that the momentum transfer it yields under the straight line assumption is approximately equal to the momentum transfer calculated for the original force using rigorous two-body orbits. The fictitious force differs substantially from the true one only at distances less than the impact parameter for $90^{\circ}$ scattering, distances much smaller than the mean separation between stars.

It should be mentioned that by dropping the three-particle correlations one necessarily ignores the formation and disruption of binaries in close, three-body encounters. 


\section{Conclusion}

The theory outlined here has not yet been used to provide accurate numerical estimates of collisional relaxation in stellar systems. It has, however, provided considerable physical insight into the dynamical processes involved. It seems likely that with further development it will turn into a useful calculational tool. 